\title{
Non-operative Management of Blunt abdominal trauma in a Tertiary Care Hospital of Nepal
}

\author{
Ghimire , $^{1^{*}}$ Yogi $N,{ }^{2}$ Ghimire $P^{3}$ \\ ${ }^{1}$ Department of Surgery, Manipal Teaching Hospital, Pokhara, Nepal, \\ ${ }^{2}$ Mch Resident National Institute of Neurological and Allied Sciences, Bansbari, Kathmandu, Nepal, \\ ${ }^{3}$ Medical Officer, Department of Surgery, Manipal Teaching Hospital, Pokhara, Nepal
}

\section{*Corresponding Author: \\ Dr. Pradeep Ghimire MS, FRCS Ed, Department of Surgery, \\ Manipal Teaching Hospital, Pokhara \\ Email: pradeepg757@hotmail.com}

\section{Citation}

Ghimire P, Yogi N, Ghimire P. Non-operative Management of Blunt abdominal trauma in a Tertiary Care Hospital of a Developing Nation. Nepal Journal of Medical Sciences 2013;2(1):38-41.

\section{Background:}

B unt Abdominal Trauma is one of the most common causes of preventable trauma-related death. It accounts for $5-15 \%$ of all operative abdominal injuries. ${ }^{1}$ Blunt trauma is responsible for about $90 \%$ of the abdominal injuries. ${ }^{2}$ In

\section{ABSTRACT}

Background: To assess the feasibility of Non-operative management of Blunt abdominal trauma in a teaching hospital of Nepal.

Methods: A prospective observational study was carried out over a period of 3 years including 52 cases of blunt abdominal trauma in a teaching hospital in western part of Nepal. Patient and trauma characteristics of the cases, different modalities of treatment and outcomes were evaluated. All the cases were divided in 3 groups: Operative group, Non-Operative Management and Non-Operative Failure group. Operative group and non-operative management group were compared using Fischer Exact Test for categorical variable and student's " $t$ " test for continuous variable.

Results: There were $36 \%$ of cases in operative group, $61 \%$ in nonoperative management group and $2 \%$ in non-operative management failure group. Non-operative management was successful in 97\% of cases. Injury severity score, admission hematocrit and hemodynamic status were significantly different between non-operative management and Operative group. Non-operative management failure occurred in 1 case and was secondary to delayed hepatic hemorrhage.

Conclusion: Non-operative management of Blunt abdominal trauma can be attempted with high degree of success. Hemodynamic and clinical instability rather than severity of the organ injury is the predictor of failure in non-operative management. Spleen and bowel injury are the most common organ that usually land up in operative group because of hemodynamic instability in splenic injury and peritoneal contamination in bowel injury. Close surveillance in an intensive care unit is always desirable.

Keywords: Non-operative Management; blunt abdominal trauma; liver trauma; spleen trauma

children sustaining trauma by blunt mechanisms, up to $8 \%$ will have injuries to the abdomen, primarily involving the solid viscera. ${ }^{1}$ During the past two decades, management of blunt injury to abdominal organs has shifted from operative to selective non-operative management. ${ }^{3}$ With the advent 
of improved intensive care, interventional radiology and trauma protocols, it is reported that blunt injuries can now be managed non-operatively with a success rate of almost $85 \% .{ }^{4}$ Current literature shows that between $50 \%$ and $98.5 \%$ of patients with blunt hepatic injury receive nonoperative management, and failure rates range from $3 \%$ to $15 \%$. Reported success rates for non-operative management of blunt splenic injury are $95 \%$ or higher for pediatric patients and approximately $80 \%$ or higher in adults. ${ }^{3}$ This observational study was carried out in a tertiary care teaching hospital of a developing nation to assess the feasibility of non-operative management of Blunt abdominal trauma.

\section{Methods:}

This is a prospective observational study done over a period of 3 years in Manipal Teaching Hospital, a tertiary care centre in western part of Nepal during the period from Jan, 2008 to Feb 2011. Fifty two patients with blunt injury to the abdomen were included in the study.

All patients of Blunt abdominal trauma were assessed by Emergency duty doctor and then attended by surgical team. After primary survey, necessary steps were taken for resuscitation according to Advanced Trauma life Support (ATLS) guidelines. ${ }^{5}$

After resuscitation patients were categorized as hemodynamically stable or unstable according to their systolic blood pressure, pulse rate, respiratory rate and admission hematocrit. All unstable patients were rushed to operation theatre without any delay. Those patients who were hemodynamically and clinically stable after resuscitation were subjected to further investigations. Routine ultrasound was done in all cases whereas CT scan of abdomen and pelvis was done only in those who had diagnostic dilemma following ultrasound. Those patients who died during the course of resuscitation in the emergency department were excluded from the study. All patients who were hemodynamically and clinically stable following resuscitation were chosen for non-operative management group. These patients were admitted to the surgical intensive care unitand had regular physical examinations along with hematocrit. Radiological evaluation was repeated whenever in doubt. Those patients who deteriorated were taken up for surgery and were termed non-operative management failure group. Decision to operate on the cases that were kept under non-operative management group was taken by the surgeon in charge of the unit.

Data were recorded for patient characteristics (age, sex) and trauma characteristics (mode of injury, hemodynamic status, organs injured, injury grading, hospital stay etc).
Trauma severity was evaluated according to Injury Severity Score (ISS) and organ injury according to Injury Scaling and Scoring System. ${ }^{6}$

Data were collected for various patient and trauma characteristics. Data were evaluated using SPSS 11.5 for windows. Operative group and non-operative management group were compared using Fischer Exact Test for categorical variable and student's " $t$ " test for continuous variable.

\section{Results:}

The total number of the patients in this study was fifty two of which $19(36 \%)$ patient underwent operative intervention and $32(61 \%)$ patients were managed non-operatively. There was $1(2 \%)$ failure in non-operative management. There were 35 (67\%) male and 17 (33\%) female. Most of the patients were young adults (21-40 years) with mean age being 27.12 years and age ranging between 4-75 years. No significant difference was noted in terms of age and sex between the operative management group and nonoperative management group. The commonest cause of blunt abdominal trauma (75\%) was Road traffic accident (RTA). The mean ISS in operative management group was 19.5 and non-operative management group was 12.4. In operative management group there was significantly higher number of cases secondary to RTA and their mean ISS was also significantly higher. There was no significant difference between the two groups in terms of injury related to fall or sports. Similarly there were significantly fewer cases with hypotension and no case with tachycardia in non-operative management group.

\section{Table 1: Patient characteristics on admission}

\begin{tabular}{lccc}
\hline & $\begin{array}{c}\text { Operative } \\
\text { Group }\end{array}$ & $\begin{array}{c}\text { Non-operative } \\
\text { managementgroup }\end{array}$ & $\begin{array}{c}P \\
\text { value }\end{array}$ \\
\hline MeanAge(Range) & $30.05(6-73)$ & $25.84(4-75)$ & 0.37 \\
Male sex & $11(58 \%)$ & $23(72 \%)$ & 0.36 \\
Mode of Injury & & & \\
RTA & $10(53 \%)$ & $28(87 \%)$ & 0.009 \\
Fall Injury & $5(26 \%)$ & $3(9 \%)$ & 0.13 \\
Sports Related & $4(21 \%)$ & $1(3 \%)$ & 0.06 \\
Mean ISS (Range) & $19.15(8-29)$ & $12.41(4-25)$ & $<0.001$ \\
Mean Hct* & $29.89(21-44)$ & $41.13(32-47)$ & $<0.001$ \\
SBP >90 mmHg* & $3(16 \%)$ & $28(87 \%)$ & $<0.001$ \\
Pulse $<100^{*}$ & 0 & $32(100 \%)$ & $<0.001$ \\
\hline
\end{tabular}

*Values from emergency department

Out of 19 cases of operative management group, liver and 
spleen were injured in 37\% and hollow viscus (bowel) was injured in $53 \%$ of cases.

\section{Table 2: Injury Characteristics}

\begin{tabular}{lccc}
\hline $\begin{array}{c}\text { Organ } \\
\text { Involved }\end{array}$ & OperativeGroup & $\begin{array}{c}\text { Non Operative } \\
\text { Group }\end{array}$ & P value \\
\hline Liver & $7(37 \%)$ & $13(41 \%)$ & 1 \\
Spleen & $7(37 \%)$ & $1(3 \%)$ & 0.003 \\
Kidney & 0 & $3(9 \%)$ & 0.28 \\
Bowel & $10(52.63 \%)$ & 0 & $<0.001$ \\
\hline
\end{tabular}

Splenectomy was done in 6 cases and repair of liver laceration in 3 cases. Out of 10 cases of bowel injury, colon was injured in 1 case, stomach in 2 cases and small intestine in rest of the 7 cases. Colostomy was required in 1 case and resection and anastomosis in 3 cases of small bowel perforation whereas rest were repaired primarily. There were significantly higher numbers of spleen and bowel injuries in operative management group whereas liver and kidney injuries showed no significant difference between the two groups.

Of 32 cases who underwent successful non-operative management, liver was injured in $13(41 \%)$ cases, spleen in $1(3 \%)$ case, kidney in $3(9 \%)$ of cases whereas in 5 cases (16\%) both ultrasound and CT scan showed evidence of minimal hemoperitoneum without any features of solid organ injury. The grade of injury of individual organs according to ISS system is illustrated in Figure 1.

Non-operative management failed in 1 patient with grade $\mathrm{V}$ liver injury. He was operated on $6^{\text {th }}$ day of admission due to hemodynamic instability. There was no mortality in our series.

\section{Discussion:}

Non-operative management of Blunt abdominal trauma is not a novelty. Over the past few decades it has become well established and strategies based on hemodynamic stability and CT scan findings are now being widely used. Even the patients with hemoperitoneum, altered mental status, higher grades of injury and older ages have now been routinely managed non-operatively in many well established trauma centers with very few failures ${ }^{7}$ but in a hospital where facilities are limited, it often becomes the challenge.

The trend in favor of non-operative management of solid organ injuries has been clearly shown by studies of blunt hepatic, splenic, and renal injuries, and further aided by the increasing availability and accuracy of various advanced imaging techniques and patient monitoring technologies. ${ }^{8,9}$ At times non-operative management of trauma may be associated with risks of missing hollow visceral injuries, delayed bleeding and transfusion related risks but then it also results in a sharp decline in the rate of both therapeutic and non-therapeutic laparotomies.

The decision to choose a patient for non-operative management should be based on hemodynamic and clinical status of the patient and not the severity or grade of organ Injury. ${ }^{10}$ Any patient with peritonitis and/or hemodynamic instability should immediately directed to the operation theatre.

If the decision has been made to observe the patient and to pursue non-operative management, close monitoring of vital signs and frequently repeated physical examinations are instituted. Adjunctive laboratory testing, such as serial determination of white blood cell count, hemoglobin and hematocrit levels, and serum lactic acid level and base deficit can also help determine if the non-operative approach is failing. The development of peritonitis on physical examination and lack of response to non-operative management, constitute an indication for surgery. In our series there was significant difference $(\mathrm{P}<0.001)$ in hemodynamic status at presentation, the admission haematocrit and the injury severity score between operative and non-operative management group.

Associated organ involvement is another decisive factor in non-operative management success. Splenic trauma has the highest failure rates reaching $30 \%{ }^{3,11}$ Splenic trauma was present in significantly higher number of cases $(37 \%)$ in operative management group than in non-operative management group (3\%). The only case of splenic injury in non-operative management group was Grade II in severity whereas there were 1, 4 and 2 cases of Grade I, II and III splenic injury in the operative management group. Evidences suggest even very severe splenic injuries, associated with significant hemoperitoneum, have been successfully managed nonoperatively. ${ }^{12}$ Splenic injury that rapidly stabilizes with little fluid or blood replacement is successful in $80-90 \%$ of cases. ${ }^{12}$

Liver has proven to be a sturdy and durable organ as the vast majority of the cases are being treated conservatively. Liver was injured in $40 \%$ of cases of non-operative management and $37 \%$ of operative management group in this study $(\mathrm{P}=1)$. The only case of failure in non-operative management in this study was secondary to a hepatic laceration grade $\mathrm{V}$ who became hemodynamically unstable on the $6^{\text {th }}$ day of admission. Some author stress that grade IV and V liver 
injuries are often associated with high morbidity (21\% and $63 \%$, respectively). The majority of such complications as ongoing bleeding, biloma, bile peritonitis, abscess or fistulae can be successfully treated with selective angioembolism, percutaneous drainage, ERCP and other minimally invasive procedures. ${ }^{13,14}$ In our study none of the patients encountered any such problems.

Similarly Non-operative management has also been applied in Kidney as well as bowel injuries. Non-operative management of renal trauma (grades I to III) has become standard. If the injury is properly staged, non-operative management is successful for contusions, contained lacerations, and even lesions with moderate amount of extravasation of urine or blood in the hemodynamically stable patient. In case renal arterial thrombosis, nonoperative management may be attempted in a stable case but with a clear understanding that late nephrectomy can occur. Non-operative management of the blunt trauma involving the bowel may be attempted in case of isolated contusions only. There are reports of successful management of duodenal injury in 57\% of cases. ${ }^{15}$ Similar approach may be applied for bowel injury provided patient is stable and has localized bowel wall hematoma or contusion on CT scan. Patient should be frequently reassessed clinically and radiologically and if any evidence of peritonitis or worsening of clinical signs, the patient should be transferred to the operating room. In our case all the 10 cases of bowel injury were operated because they all had perforation at various sites.

\section{Conclusion:}

In our study non-operative management was successful in $97 \%$ of cases. Non-operative management of Blunt abdominal trauma can be attempted with high degree of success. Hemodynamic and clinical instability rather than severity or grade of the organ injury is the predictor of failure in non-operative management. Spleen and bowel injury are the most common organ that usually land up in operative group because of hemodynamic instability in splenic injury and peritoneal contamination in bowel injury. Close surveillance of such patient in an intensive care unit is always desirable.

\section{Conflict of interest: nil}

\section{References:}

1. Keller MS. Blunt injury to solid abdominal organs. Semin Pediatr Surg 2004;13:10611.

2. Leung E, Wong L, Taylor J. Non-operative management for blunt splenic trauma in children: An updated literature review. Surgical Practice 2007;11:29-35.

3. Yanar H, Ertekin C, Taviloglu K, et al: Nonoperative treatment of multiple intra-abdominal solid organ injury after blunt abdominal trauma. J Trauma 2008;64:94348.

4. Thompson SR, Holland AJ. Current management of blunt splenic trauma in children. ANZ J Surg 2006;76:48-52.

5. American College of Surgeons: ATLS Advanced Trauma Life Support Program for Doctors. Chicago, IL, USA 7th edition. 2004.

6. AAST Injury Scaling and Scoring System. Trauma Tools - The American Association for the Surgery of Trauma

7. Schwab CW. Selection of non-operative management candidates. World J Surg 2001;25:1389-92.

8. Knudson MM, Maull KI. Non-operative Management of solid organ injuries- Past, Present and Future. Surg Clin North Am 1999;79:1357-71.

9. Christmas AB, Wilson AK, Manning B, et al. Selective management of blunt hepatic injuries including nonoperative management is a safe and effective strategy. Surgery 2005;138:606-10.

10. McConnell DB, Trunkey DD. Non-operative management of abdominal trauma. Surg Clin North Am 1990;70:677-88.

11. Cadeddu M, Garnett A, Al-Anezi K, et al: Management of spleen injuries in the adult trauma population: a tenyear experience. Can J Surg 2006;49:386-90.

12. Meredith JW, Young JS, Bowling J, et al. Non-operative management of blunt hepatic trauma: the exception or the rule. J Trauma 1994;36:529-35.

13. Velmahos GC, Toutouzas K, Radin R, et al: High success with non-operative management of blunt hepatic trauma: the liver is a sturdy organ. Arch Surg 2003;138:475-80.

14. Kozar RA, Moore FA, Cothren CC, et al: Risk factors for hepatic morbidity following nonoperative management: multicenter study. Arch Surg 2006;141:451-8.

15. Huerta S, Bui T, Porral D, et al. Predictors of morbidity and mortality in patients with traumatic duodenal injuries. Am Surg 2005;71:763-67. 